\title{
Die Zukunft des Gesundheitswesens
}

\author{
Ziel: Primärversorgung und Zusammenarbeit der Gesundheitsberufe stärken
}

Veränderte Bevölkerungsstrukturen, neue Kompetenzen der Gesundheitsund Krankenpflege und gesetzliche Vorgaben, die bisweilen schwer umzusetzen sind - das österreichische Gesundheitssystem funktioniert in seinen traditionellen Bahnen nicht immer optimal. Mitarbeiter und Patienten aber auch die politisch Verantwortlichen sind mit dem Ergebnis nicht immer zufrieden. „Die Zukunft des Gesundheitswesens" stand beim 9. Pflege-Management Forum Anfang März in Wien im Mittelpunkt einer Diskussion von Repräsentanten der unterschiedlichen Akteure.

„Der Patient steht im Mittelpunkt, aber dort steht er im Weg", formulierte Ursula Frohner, Präsidentin des Österreichischen Gesundheits- und Krankenpflegeverbands (ÖGKV) pointiert einen Teil des Dilemmas im österreichischen Gesundheitssystem. Er kommt zum falschen Zeitpunkt, hält sich oft nicht an die Empfehlungen oder Anordnungen der Gesundheitsmitarbeiter, ist ängstlich, aufmüpfig, manchmal auch aggressiv und kostet insgesamt oft viel Geld. Vieles ließe sich durch eine gezieltere $\mathrm{Zu}$ wendung und mehr Zeit wesentlich verbessern. Doch das scheitert derzeit noch allzu oft - aus strukturellen, standespolitischen und durchaus auch persönlichen Gründen. Denn in der Zusammenarbeit der Berufsgruppen rund um den „Fokus Patient“ hapert es nach wie vor.

\section{Kein Dialog auf Augenhöhe für die Pflege}

Ziel sollte sein, die Primärversorgung zu stärken und alle Gesundheitsberufe gemeinsam arbeiten zu lassen, wie Eva-Maria Kernstock von der Gesundheit Österreich (GÖG) feststellte. Allerdings haben, so Frohner, 65 Prozent der in Gesundheitsberufen Arbeitenden kein Mitspracherecht: Die Gesundheits- und Krankenpflege. Der Dialog auf Augenhöhe bei politischen Entscheidungen findet für die Gesundheitsund Krankenpflege nicht statt. Positive Signale kamen diesbezüglich jedoch von Mag. Martin Schaffenrath vom Hauptverband der Sozialversicherungsträger, einem der Hauptverhandlungspartner in Sachen Gesundheitsreform: „Ich würde mir wün-

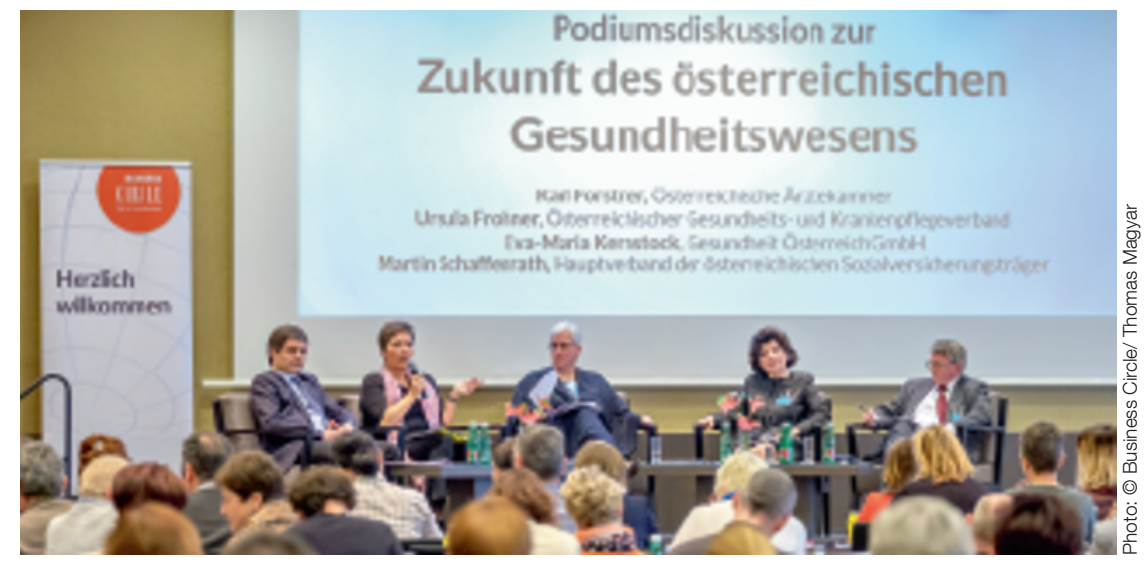

schen, wenn die Pflege mit am Tisch sitzt. Ich unterstütze das!“

Einig waren sich jedenfalls alle Diskussionsteilnehmer, dass die Gesundheitsversorgung neu strukturiert werden muss: Weg von der Spitalslastigkeit, wie Dr. Karl Forstner von der Österreichischen Ärztekammer konstatierte, hin zu einer Stärkung der Primärversorgung. Dass diese nicht immer in Form von Zentren, sondern auch über das Instrument der Vernetzung erreicht werden kann, unterstrichen sowohl Frohner als auch Schaffenrath. Dafür aber werden neue Organisationsformen notwendig sein, die, so Frohner „rasch erarbeitet werden müssen“. Dass der gehobene Dienst für Gesundheits- und Krankenpflege dabei eine wesentliche Rolle spielen wird, ist für die Berufsvertretung eine notwendige Entwicklung (siehe auch Interview auf Seite 4 dieser Ausgabe). Schon heute übernimmt der gehobene Dienst einen Anteil an medizinischen Versorgungsleistungen.

Von Seiten der Sozialversicherung und der Ärztekammer sieht man freilich weiterhin den Arzt als Drehscheibe in der Primärversorgung und die Ordinationen als Anlaufstelle für Patienten vor allem im ländlichen Raum. Es werde, so Kernstock, viel Unterstützung brauchen, bis eine Umsetzung der Vorgaben durch die Gesundheitsreform tatsächlich stattfindet: „Die Reformgedanken müssen erst bei den Berufsgruppen selbst ankommen." Während die eine Berufsgruppe ihre tradierten Verantwortlichkeiten nicht abgeben oder teilen möchte, hat die andere Probleme, diese selbstbewusst einzufordern. „Die
Gesundheits- und Krankenpflege muss sich ihrer Bedeutung bewusst sein", forderte Frohner. Nach aktuellen Berechnungen werden im Jahr 2013 in Österreich 20.000 Pflegekräfte fehlen - mit einer großen Bandbreite an Aufgaben.

\section{Gesundheitsreform auch für die Mitarbeiter}

Die Gesundheitsreform soll jedenfalls auch den Mitarbeitern selbst zugute kommen, betonte Kernstock. Beispielsweise was die Karriereplanung angeht, denn damit werde es möglich, zu erheben, welche Berufsgruppen mit welcher Qualifikation über einen bestimmten Zeitraum benötigt werden. Eine Angleichung der Leistungen der Sozialversicherungen werde dagegen weiterhin schwierig, stellte Schaffenrath fest, wenngleich klar sei, dass „irgendwann eine Leistungsharmonisierung gefunden werden muss.“ Aber: „Gleiche Leistung bei gleichem Geld wird viel Geld kosten." Die Anforderungen an eine Umstrukturierung des Gesundheitssystems sind also hoch und sie muss umfassend sein. „Die Gesundheitsreform ist am richtigen Weg“, meinte Forstner aus Sicht der Ärztekammer, allerdings: „Nur an Organisationsformen herumzubasteln wird nichts nützen." Auch darin herrschte bei den Beteiligten grundsätzlich Einigkeit. Wenn auch vielleicht mit unterschiedlichen Ansichten über die Ausrichtung. 